\title{
An Efficient Algorithm Based on Power-Kepstrum to Reduce Multipath Interference of Microwave Landing System
}

\author{
Yarong Chen ${ }^{\mathrm{a}}$, Wenzhun Huang ${ }^{\mathrm{b}}$, \\ Wenjun $\mathrm{Huo}^{\mathrm{c}}$, Jiang $\mathrm{Liu}^{\mathrm{b}}$ and Jiahao Wang ${ }^{\mathrm{b}}$ \\ (Dept of Information Engineering, Xijing University, Xian 710123, China) \\ accyyrr1029@sina.com, bhuangwenzhun@xijing.edu.cn, 9huowj_2008@163.com
}

Keywords: Microwave landing system; Time reference scanning beam; Multipath interference; Power-cepstrum; Time delay estimation

\begin{abstract}
The multipath interference affects the navigational accuracy of the Microwave Landing System (MLS), which threatens the landing security of planes seriously. The common methods of the multipath interference suppression are realized on the electromagnetic modeling of the airport environment, which have huge computation and complicated design, etc. A novel method based on the power-cepstrum technology is proposed to restrain the multipath signal of MLS. Firstly, the mathematical model of the signals received by the navigational equipments on the plane. And then, the power-cepstrums of the signals are calculated and the multipath effect is counterbalanced so that the time delay between the toward-and-back scanning beams is estimated precisely. Finally, its effectiveness is proved by the simulation and experiment.
\end{abstract}

\section{Introduction}

Microwave Landing System is an advanced land-based landing system recommended by the International Civil Aeronautics Organization (ICAO). Compared with the Instrument Landing System (ILS), the MLS enables much wider coverage, and enables conduct of area navigation in the vicinity of the airport. These new capabilities make the MLS to better meet the requirements of modern aviation. But like to other systems of wireless measurement, there is some inaccuracy in the MLS avionics ineluctable. Especially, the multipath effect resulted from the complicated electromagnetic environment around the airport reduces the navigational accuracy of the MLS, which threatens the landing security of the approaching airplanes seriously.

To reduce the effect of the multipath interference, the paper [4] and the paper [4][5] established the model of electromagnetic scatter to simulate the potential multipath obstacles. Based on the estimation of the multipath error on the airport site, the paper [6] adjusted the aperture of the scan antenna on the ground to depress the influence of the multipath. But, the above-mentioned methods have the problems of huge computation and complicated design. In this paper, a method, based on the process of digital signal, is proposed to estimate the time delay between two scanning signals directly by the power-cepstrum. Therefore, the ability of multipath restraint is improved obviously in order to attain the accurate space position of the approaching airplane.

\section{Mathematical Model of the MLS receiving Signals}

Navigation principle of the MLS. The MLS uses the technique named time reference scanning beam (TRSB) to observe azimuth angle, elevation angle and distance measuring equipment, so that to determine the aircraft position. In the azimuth guidance of the MLS, for example, an azimuth antenna located near the stop-end of a runway, generates a narrow, flabellate beam which provides continuous to-and-fro scanning in an invariable speed within the MLS lateral coverage. The aircraft avionics receives two pulse signals shaped by the to-and-fro scanning, and measures the time delay between those two signals to compute the azimuth angle of the landing airplane relative to the middle line of a runway. The principle of the azimuth angle measurement in the MLS is illustrated in Fig. 1, similar to that of the elevation guidance. 


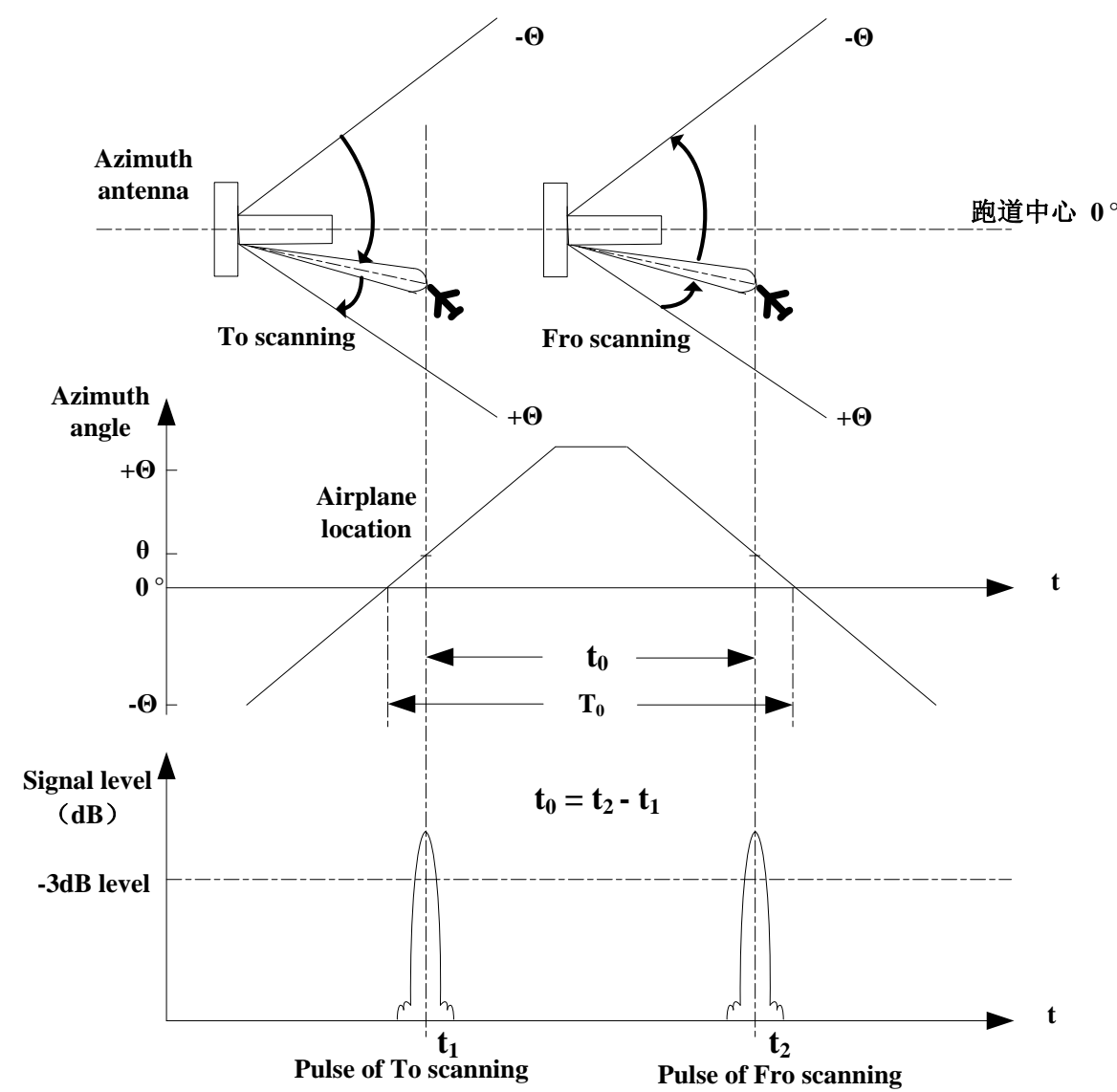

Figure 1. Principle of the azimuth angle measurement in the MLS

As shown in Figure 1, if the time delay $t_{0}$ of the two pulse signals received by the aircraft avionics is measured, then its azimuth angle can be computed by Eq. 1:

$$
\theta=\frac{1}{2}\left(T_{0}-t_{0}\right) \cdot v
$$

Where, $\theta(\mathrm{rad})$ is the azimuth angle undetermined; $T_{0}(\mathrm{~ms})$ is a design constant, means the time interval of the to-and-fro scanning which passes through the middle line of a runway where the azimuth angle is $0^{\circ} ; t_{0}(\mathrm{~ms})$ is the time delay between two received pulse signals; $v(\mathrm{rad} / \mathrm{ms})$ is $20(\mathrm{rad} / \mathrm{ms})$, means the velocity of scanning. 
Data Model for Navigational Signals Disturbed by Multipath Errors. When there is no interference of the multipath, the scanning signal received by the avionics shapes to a symmetrical and raised waveform, which has only one maximum peak point. The offset from the ideal landing course, which can provide a navigation fix or route to the pilot, can be computed by the time interval $t_{0}$ between the two peaks. But under the influence of the geographical environment of the airport and the large buildings near the runway, illustrated in Fig. 2, the raised waveform of the scanning signal is distorted by the multipath signals so that the avionics in the aircraft can not calculated the azimuth angle correctly.

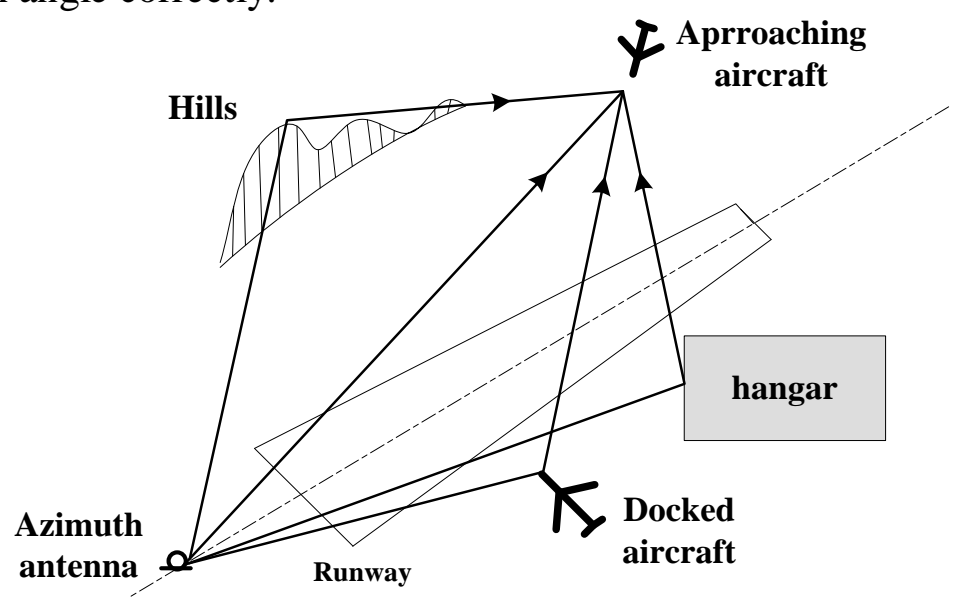

Figure 2. Illustration of signals propagation with multipath interference

Consider the signal transmitted by a ground azimuth antenna is $s(t)$, the two scanning signal received in the aircraft $x_{1}(t)$, is disturbed by M multipath signals, then:

$$
\begin{aligned}
x_{1}(t) & =s(t)+\sum_{i=1}^{M} A_{i} s\left(t-\tau_{i}\right)+n_{1}(t) \\
& =s(t) *\left[\delta(t)+\sum_{i=1}^{M} A_{i} \delta\left(t-\tau_{i}\right)\right]+n_{1}(t)
\end{aligned}
$$

Where $\tau_{i}$ denotes the time delay between the $i$ th multipath signal and the direct signal, $A_{i}$ denotes the fading factor of the $i$ th.

If $h(t)$ is written as

$$
h(t)=\delta(t)+\sum_{i=1}^{M} A_{i} \delta\left(t-\tau_{i}\right)
$$

Then

$$
x_{1}(t)=s(t) * h(t)+n_{1}(t), \quad 0 \leq t<T
$$

Suppose the instantaneous model of the multipath interference unchanged, the fro scanning signal received by the avionics, $x_{2}(t)$, can be written as

$$
x_{2}(t)=s\left(t-t_{0}\right) * h(t)+n_{2}(t), \quad 0 \leq t<T
$$

Discrete (4) and (5), then:

$$
\left\{\begin{array}{l}
x_{1}(n)=s(n) * h(n)+n_{1}(n) \\
x_{2}(n)=s\left(n-n_{0}\right) * h(n)+n_{2}(n)
\end{array}, \quad 0 \leq n \leq N-1\right.
$$

Where

$$
T_{s}=T / N, \tau_{i}=n_{i} T_{s}, t_{0}=n_{0} T_{s}
$$

$T$ is the time of the observation duration, $T_{s}$ is the sampling interval.

To simplify the description of the problem, suppose SNR of the received signals is so high that 
the additive noise can be ignored. Then Eq. 6 has the form

$$
\left\{\begin{array}{l}
x_{1}(n)=s(n) * h(n) \\
x_{2}(n)=s\left(n-n_{0}\right) * h(n)
\end{array}, \quad 0 \leq n \leq N-1\right.
$$

The waveform of the single is illustrated in Figure 3, in which the blue curve describes the signal waveform without multipath interference and the red curve describes that with multipath interference. From Fig. 3 it can be noted that the received signal waveform is distorted by the multipath error so that the peak time $t_{1}$ of the direct wave can not be observed exactly and $t_{0}$ can not be calculated accurately consequently.



Figure 3 Waveform of the received signal

\section{Multipath Error Suppression Algorithm Based on Power-Cepstrum}

From Eq. 7, we can see that the scanning signal received by the aircraft avionics is formed with the convolution of the direct signal transmitted from the ground azimuth antenna and the multipath channel. According to the theory of digital signal processing, the method based on the cepstrum is propitious to deconvolution to separate multiplicative noise. Therefore, the multipath interference in the MLS can be regarded as the multiplicative noise, which can be suppressed by the cepstrum method, and $t_{0}$ can be estimated accurately.

Firstly, construct two new sequences $x_{3}(n)$ and $x_{4}(n)$ according to the to-and-fro sequences received by the aircraft avionics:

$$
\left\{\begin{array}{l}
x_{3}(n)=x_{1}(n)+x_{2}(n) \\
x_{4}(n)=x_{1}(n)-x_{2}(n)
\end{array}, \quad 0 \leq n \leq N-1\right.
$$

Then, calculate the Discrete Fourier Transformation (DFT) of the above two sequences, written as $X_{3}(k)$ and $X_{4}(k)$ :

$$
\left\{\begin{array}{l}
X_{3}(k)=X_{1}(k)+X_{2}(k)=S(k) \cdot H(k) \cdot\left(1+e^{-j \frac{2 k \pi}{N} \cdot n_{0}}\right) \\
X_{4}(k)=X_{1}(k)-X_{2}(k)=S(k) \cdot H(k) \cdot\left(1-e^{-j \frac{2 k \pi}{N} \cdot n_{0}}\right)
\end{array} \quad, \quad 0 \leq k \leq N-1\right.
$$

Evaluate the logarithm of $X_{3}(k)$ and $X_{4}(k)$ :

$$
\ln X_{3}(k)=\ln S(k)+\ln H(k)+\ln \left[1+e^{-j \frac{2 k \pi}{N} \cdot n_{0}}\right], \quad 0 \leq k \leq N-1
$$




$$
\ln X_{4}(k)=\ln S(k)+\ln H(k)+\ln \left[1-e^{-j \frac{2 k \pi}{N} \cdot n_{0}}\right], \quad 0 \leq k \leq N-1
$$

After calculating the difference of Eq. 10 and Eq. 11 to counteract the common items $\ln S(k)$ and $\ln H(k)$, we can have $\ln X(k)$ as:

$$
\ln X(k)=\ln X_{3}(k)-\ln X_{4}(k)=\ln \left[\frac{1+e^{-j \frac{2 k \pi}{N} \cdot n_{0}}}{1-e^{-j \frac{2 k \pi}{N} \cdot n_{0}}}\right], \quad 0 \leq k \leq N-1
$$

From power series expansion theory, we know that:

$$
\ln \left[\frac{1+x}{1-x}\right]=2 \sum_{l=1}^{\infty} \frac{x^{2 l-1}}{2 l-1}=2 x+\frac{2}{3} x^{3}+\frac{2}{5} x^{5}+\cdots
$$

Therefore, the right of Eq. 12 can be expanded as:

$$
\ln X(k)=2 e^{-j \frac{2 k \pi}{N} \cdot n_{0}}+\frac{2}{3} e^{-j \frac{2 k \pi}{N} \cdot 3 n_{0}}+\frac{2}{5} e^{-j \frac{2 k \pi}{N} \cdot 5 n_{0}}+\cdots, \quad 0 \leq k \leq N-1
$$

Through Inverse Discrete Fourier Transform of $\ln X(k)$, we have

$$
\tilde{x}(n)=2 \delta\left(n-n_{0}\right)+\frac{2}{3} \delta\left(n-3 n_{0}\right)+\frac{2}{5} \delta\left(n-5 n_{0}\right)+\cdots
$$

From Eq. 15 it can be noted that, $\tilde{x}(n)$ in the cepstrum has the maximum value at $n=n_{0}$, and has decreasing amplitude peak values by $2 n_{0}$ interval. Thus, we can search for the point $n=n_{0}$ in the cepstrum where is the maximum value position of $\tilde{x}(n)$. At last, the azimuth position of the approaching airplane can be estimated exactly:

$$
\theta=\frac{1}{2}\left(T_{0}-n_{0} \cdot T_{s}\right) \cdot v
$$

\section{Simulation Results}

The effectiveness of the multipath error suppression algorithm based on power-cepstrum is verified via simulation experiments in this section.

Suppose the scanning signal received by the aircraft avionics is simulated by the main lobe of bell-shaped pulse, which could be written as:

$$
s_{i}(t)=S a\left[\alpha\left(t-\tau_{i}\right)\right]=\left\{\begin{array}{cc}
1 & t=\tau_{i} \\
\frac{\sin \pi \alpha\left(t-\tau_{i}\right)}{\pi \alpha\left(t-\tau_{i}\right)} & t \neq \tau_{i}
\end{array}\right.
$$

Where $\alpha$ contents

$$
\left.S a[\alpha t]\right|_{t=\frac{275}{2} \mu s}=\frac{\sqrt{2}}{2}
$$

The observation time of the to-and-fro signals is $12 \mathrm{~ms}$, which simulates the slot time application of the azimuth scanning in the MLS. The sampling frequency is $10 \mathrm{MHz}$ (the sampling interval $\left.T_{s}=0.1 \mu \mathrm{s}\right)$. The simulating waveforms in time domain without additive noise are illustrated in Fig. 4. Fig. 4(a) shows the signal waveform without multipath interference and Fig. 4(b) shows the signal waveform with four multipath interference, where the multipath time delays are $\tau_{1}=23 \mu s$, $\tau_{2}=40 \mu \mathrm{s}, \tau_{3}=212 \mu \mathrm{s}, \tau_{4}=613 \mu \mathrm{s}$, the amplitude fading factors are $A_{1}=0.6, A_{2}=0.5$, $A_{3}=0.3, A_{4}=0.1$. 
(a) signal waveform without multipath interference

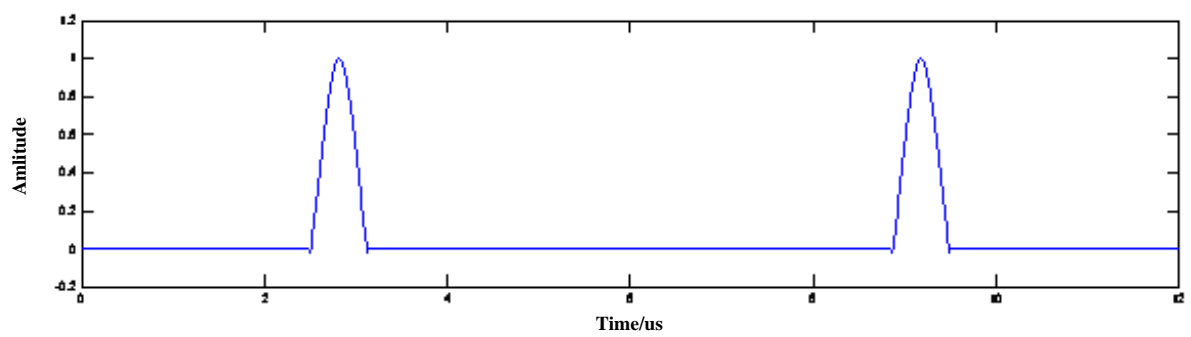

(b) signal waveform with 4-multipath interference

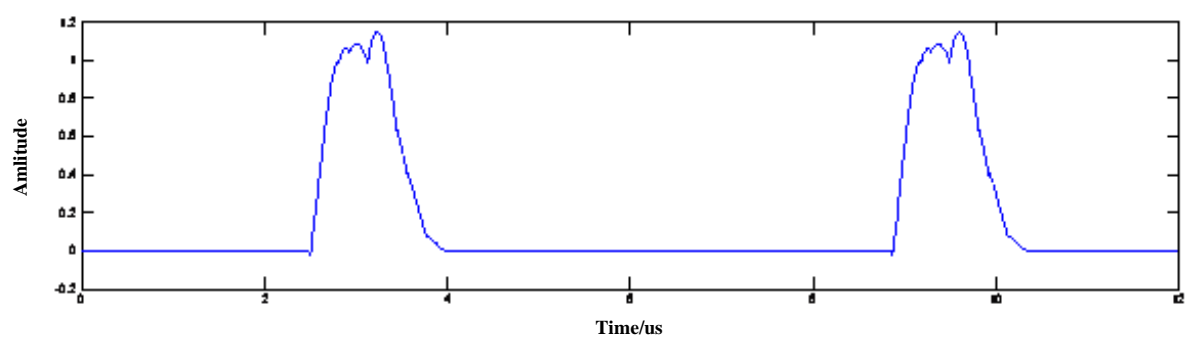

Figure 4 Simulating waveforms of the received signals in time domain

In order to verify the effectiveness of the algorithm, the composite signals are mixed into white Gaussian noise with different SNR respectively. Fig. 5 gives the time delay $t_{0}$ with different SNR $(20 \mathrm{~dB}, 10 \mathrm{~dB}$ and $0 \mathrm{~dB})$ calculated according to the algorithm in part 3.
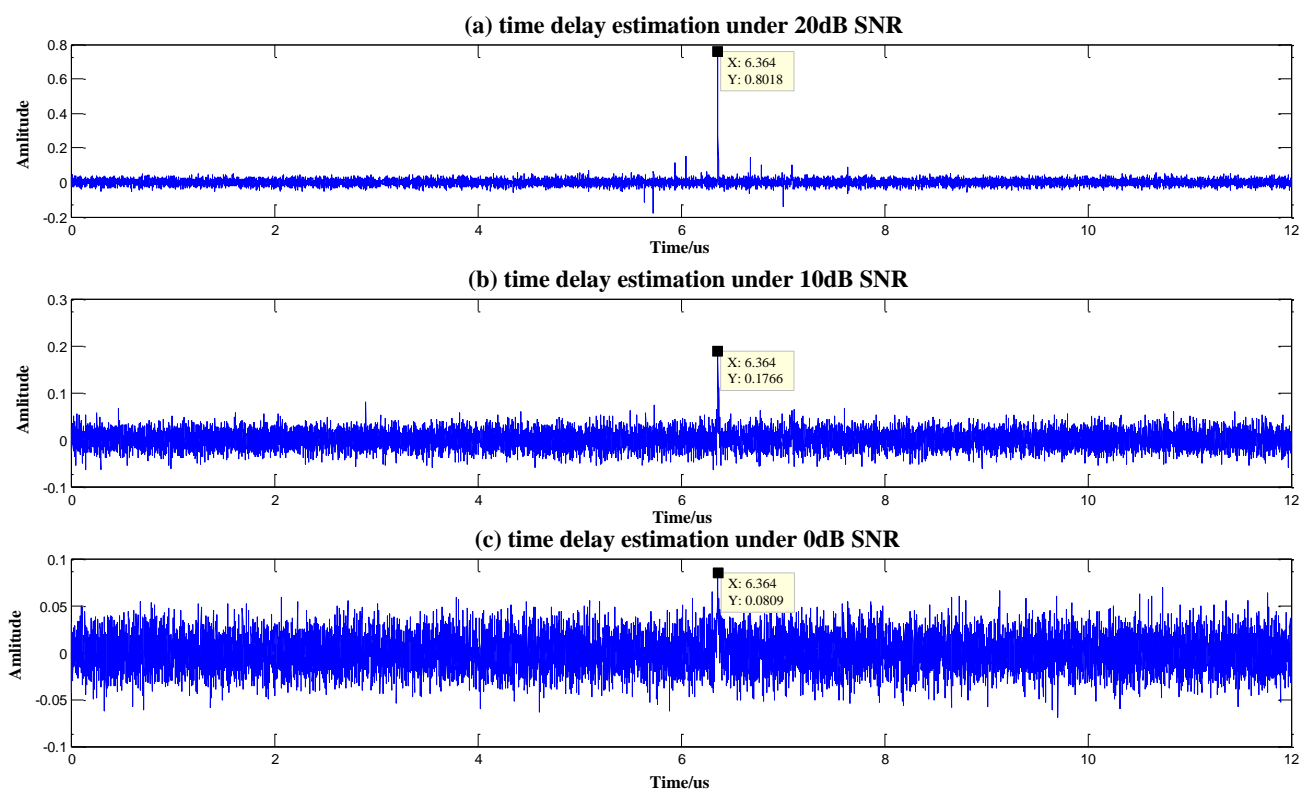

Figure 5 Estimation of the time delay under different SNR

From the results of the simulation, we can see that there is still a peak value near the real time point despite SNR decreasing. In the simulation, the real time delay $t_{0}$ is $6.365 \mathrm{~ms}$, and the estimated is $6.364 \mathrm{~ms}$, where the error of estimation is $0.15 \%$. Furthermore, calculated by Eq. 1 , the real azimuth angle is $56.35^{\circ}$, the estimated is $56.36^{\circ}$, and the error of angle measurement is $0.01^{\circ}$.

From the above simulation results it can be concluded that, the multipath error suppression algorithm based on power-cepstrum exhibits good estimation performance, and it could be recommended to be used in the MLS approaching navigation. 


\section{Summary}

The complicated electromagnetic environment around the airport leads to the multipath interference which affects the approaching navigational accuracy of the MLS distinctly. In this paper, a multipath error suppression algorithm based on power-cepstrum is proposed, which could separate the multipath interference in cepstrum and estimate the information of the approaching aircraft position precisely. The proposed method does not need to know the interference and the airport environment in advance, and has such advantages as simple algorithm and high accuracy degree. Simulation results have demonstrated the performance of the new method.

\section{References}

[1] Breien T. Computer analysis of MLS in multipath environment. Proceedings of International Conference on The Future of Aircraft All Weather Operations . 1976.

[2] Zhou Q H, Wei X Z, Cui H Y. Microwave Landing System [M].Beijing: Publishing House of National Defense Industry,1989: 101-103.

[3] Arnold, R. MLS: keeping pace with the future. Aerospace and Electronic Systems Magazine, IEEE. Volume 5, Issue 5, May 1990 Page(s): 23 - 26.

[4] Michael L, Bernhard K, Thomas J. et al. Assessment of multipath in aeronautical environments [R]. ICAO-Report, 2008.

[5] Gerving G. Statues and capabilities of advanced computer based analysis and simulations for ATV-navigation and radar system-examples [C]. HongKong, China: Proceedings of the 5th International Symposium on Electronics in the Air Transport Industry SEATI, 2006: 230-235.

[6] Dai C J, Wu D W, Zhao X B. Antenna design of microwave landing system based on the consideration of the multi-path effects [J]. Systems Engineering-Theory \& Practice, 2010, 30(11): 2099-2105.

[7] Wan J W, Wang L. Signal Processing Simulation Technology [M].Changsha: National University of Defense Technology Press, 2008: 235-237.

[8] Zhang Y, Ying X F, Tian B. An Anti-multipath-fading Algorithm Based on Cepstrum Analysis [J]. Signal Processing, 2003, 19(1):55-58.

[9] Liu J, Yang S E, Piao S C. The Signal Channel Blind Source Separation of Multipath Acoustic Signal Based on an Improved Cepstrum [C]. The Proceeding of the First National Conference on Underwater Security and Technology, 2010: 53-57.

[10] Martins E N, Sérgio M J. Blind Estimation of the Ocean Acoustic Channel by Time-freqiency Processing [J]. IEEE Journal of Oceanic Engineering, 2006, 31(3): 646-656.

[11] Azuma OKAMOTO, Mitsumasa SUGAWARA. Solving composite scheduling problems using the hybrid genetic algorithm[J]. Journal of Zhejiang University-Science A(Applied Physics \& Engineering). 2010(12).

[12] Jason Cong, Bingjun Xiao. FPGA-RPI: A novel FPGA architecture with rram-based programmable interconnects. IEEE Transactions on Very Large Scale Integration (VLSI)Systems . 2014. 